\title{
Two Vertically Coupled Quantum Rings with Tunneling
}

\author{
L. K. Castelano, G.-Q. Hai, \\ Instituto de Física de São Carlos, Universidade de São Paulo, 13560-970, São Carlos, SP, Brazil \\ B. Partoens, and F. M. Peeters \\ Department of Physics, University of Antwerp, B-2020 Antwerp, Belgium
}

Received on 8 December, 2005

\begin{abstract}
We study the effect of tunneling on the electronic structure of two vertically coupled quantum rings within the spin density functional theory. The ground state configurations of the coupled rings are obtained for a system with 10 electrons as a function of the ring radius and the inter-ring distance. For small ring radius, our results recover those of coupled quantum dots. For large ring radius, new ground state configurations are found in the strong tunneling regime.
\end{abstract}

Keywords: Quantum ring; Tunneling; Many-body effects; Ground state

\section{INTRODUCTION}

Recently, few electron self-assembled semiconductor quantum rings have been created in the nanoscopic regime [1]. This quantum structure provides an interesting system to study the ground state and the excitations in the scattering free and few-electron limit. Although theoretical studies are avaliable on single electron properties of such a confined system, a detailed study of the electron-electron interaction in the many electron case are not yet avaliable. Simonin et al. [2] have shown that, without considering electron-electron interaction, a displaced parabolic model is suitable for describing the confinement potential for a self-assembled semiconductor quantum ring. Very recently, Granados et al.[3] have shown that the formation of self-assembled quantum rings in stacked layers is possible by molecular beam epitaxy in an InGaAs semiconductor structure.

On the other hand, artificial molecules composed of coupled quantum dots (CQDs) provide an additional degree of freedom to explore new regimes of molecular physics. In these systems, interdot coupling depends not only on the electrostatic Coulomb interaction but also on the quantum tunneling between them. Tunneling strength between the coupled quantum dots can be controlled to a high precision in semiconductor growth and pattern processes. Recently, new molecular phases have been predicted and confirmed in fewelectron vertically coupled quantum dots in the intermediate coupling regime $[4,5]$. It was confirmed that the exchangecorrelation effects in the electron-electron interaction is essential to understand the new artificial molecular structures. We expect that two coupled quantum rings (CQRs) form a new type of artificial molecule where the ring radius can be a new parameter providing one more degree of freedom to modulate and control the electronic structure of the artificial ring shaped "molecule". Furthermore, due to the peculiar properties of nanoscopic rings in a magnetic field e.g. the Aharonov-Bohm effect, one can expect curious properties of quantum states in such artificial molecules formed by coupled quantum rings in the presence and in the absence of an external magnetic field.

\section{THEORETICAL MODEL}

Within the spin density functional theory, we study the ground state of few-electron two vertically coupled quantum rings. The two vertically coupled quantum rings are described by the displaced parabolic potential model $V(r)=$ $\frac{1}{2} m^{*} \omega_{0}^{2}\left(r-r_{0}\right)^{2}$ in the $x y$-plane, where $\mathbf{r}=(x, y)=(r, \theta), \omega_{0}$ is the confinement frequency and $r_{0}$ is the radius of the ring. The two stacked rings are coupled in the $z$ direction described by two coupled quantum wells with a finite barrier. The quantum wells are assumed to be $W=120 \AA$ wide with $V_{0}=250 \mathrm{meV}$ the height of the barrier between them. For these parameters we find the following expression for the energy splitting $\Delta=22.86 \exp [-d(\AA) / 13.455] \mathrm{meV}$ between the two lowest levels in the coupled quantum wells separated by a distance $d$ [4].

We use the Kohn-Sham orbitals $\psi_{n m \sigma}(\mathbf{r})=$ $\exp (-\operatorname{im} \theta) \phi_{n m \sigma}(r) Z(z)$ to express the density and ground state energy. The Kohn-Sham equation in spin density functional theory for the CQRs is given by

$$
\begin{aligned}
& {\left[-\frac{\hbar^{2}}{2 m^{*}}\left(\frac{\partial^{2}}{\partial r^{2}}+\frac{1}{r} \frac{\partial}{\partial r}-\frac{l^{2}}{r^{2}}+\frac{\partial^{2}}{\partial z^{2}}\right)+\frac{1}{2} m^{*} \omega_{0}^{2}\left(r-r_{0}\right)^{2}\right.} \\
& \left.+V(z)+V_{H}^{\text {intra }}(r)+V_{H}^{\text {inter }}(r)+\frac{\delta E_{x c}}{\delta \rho^{\sigma}(r)}\right] \phi_{n m \sigma}(r) Z(z) \\
& =\varepsilon_{n m \sigma} \phi_{n m \sigma}(r) Z(z),
\end{aligned}
$$

where $\sigma=\uparrow$ or $\downarrow$ being the $z$ component of the electron spin. The total density in the rings is $\rho(r)=\sum_{\sigma} \sum_{n, m}^{N_{\sigma}}\left|\phi_{n m \sigma}(r)\right|^{2}$. Because we are adopting two identical rings, the density in each ring is half this total density [6]. In the calculation, we approximate the density in the $z$ direction by $\delta$ functions. This approximation has been used earlier by Partoens and Peeters[4] for two coupled quantum dots and will not change our results qualitatively. The intra-ring and inter-ring Hartree potentials are given by

$$
V_{H}^{\text {intra }}(r)=\int d \mathbf{r}^{\prime} \frac{e^{2} \rho\left(r^{\prime}\right) / 2}{\varepsilon\left|\mathbf{r}-\mathbf{r}^{\prime}\right|},
$$


and

$$
V_{H}^{\text {inter }}(r)=\int d \mathbf{r}^{\prime} \frac{e^{2} \rho\left(r^{\prime}\right) / 2}{\varepsilon\left|\mathbf{r}-\mathbf{r}^{\prime}+\mathbf{d}\right|},
$$

respectively, with the inter-ring distance $d=|\mathbf{d}|$. The exchange-correlation energy functional within the local density approximation is given by $E_{x c}=2 \pi \int \rho(r) \varepsilon_{x c}\left[\rho^{\uparrow}, \rho^{\downarrow}\right]$, where the Tanatar-Ceperley [7] functional for $\varepsilon_{x c}\left[\rho^{\uparrow}, \rho^{\downarrow}\right]$ was used. The ground state energy of the coupled rings is obtained from

$$
\begin{aligned}
E(N)= & \sum_{i \sigma} \varepsilon_{i \sigma}-\frac{1}{2} \int d \mathbf{r} \rho(r)\left[V_{H}^{\text {intra }}(r)+V_{H}^{\text {inter }}(r)\right] \\
& -\sum_{\sigma} \int d \mathbf{r} \rho^{\sigma}(r) \frac{\delta E_{x c}\left[\rho^{\uparrow} / 2, \rho^{\downarrow} / 2\right]}{\delta \rho^{\sigma}(r)} .
\end{aligned}
$$

We expand the eigenfunctions $\phi_{n m \sigma}(r)$ in the Fock-Darwin[8, 9] basis to solve the Kohn-Sham equation.

We consider only the two lowest levels of the quantum wells that connect the two quantum rings in the $z$ direction. They are the symmetric bonding level and the antisymmetric antibonding level. The contribution from excited states due to confinement in the $z$ direction is neglected because the confinement in the $z$ direction is much stronger than that in the plane. Therefore the motion in the $z$ direction may be assumed to be decoupled from the in-plane motion and the Kohn-Sham equation can be solved separately. In the limits of small and large inter-ring distance $d$, the results for a single quantum ring are recovered.

\section{NUMERICAL RESULTS AND DISCUSSIONS}

First of all, we calculate the energy spectrum of a single quantum ring without electron-electron interaction. The confinement potential of the ring is taken as $V(r)=\frac{1}{2} m^{*} \omega_{0}^{2}(r-$ $\left.r_{0}\right)^{2}$ with zero thickness in the $z$ direction. The single electron states can be obtained numerically by diagonalizing the matrix Hamiltonian in the well known Fock-Darwin basis. Figure 1 shows the energy levels $\varepsilon_{n, m}$ as a function of the ring radius in unit $a_{0}=\sqrt{\hbar / m^{*} \omega_{0}}$ with the radial quantum number $n=$ $0,1,2, \ldots$, and the angular quantum number $m=0, \pm 1, \pm 2, \ldots$ . In comparison with the quantum dot $\left(r_{0}=0\right)$ results, an important difference in the single particle picture of a quantum ring is that a finite ring radius lifts the degeneracy of the states $\varepsilon_{0, \pm 2}$ and $\varepsilon_{1,0}$. With increasing the ring radius, the energy difference $\varepsilon_{1,0}-\varepsilon_{0, \pm 2}$ increases and tends to a constant for large $r_{0}$. This indicates that, in the absence of external fields, the ground state electronic structure of a quantum ring can be distinctly different from that of a quantum dot. This effect becomes apparent only for a system with 9 electrons or more when the energy levels $\varepsilon_{1,0}$ and $\varepsilon_{0, \pm 2}$ become occupied. This is also true for two coupled quantum rings. Notice that the low lying energy levels exhibit a minimum as a function of the ring radius for the $m=0$ orbitals. The reason is that, when the ring radius increases, a transition from the quantum dot to a quantum ring takes place. This effect can be understood

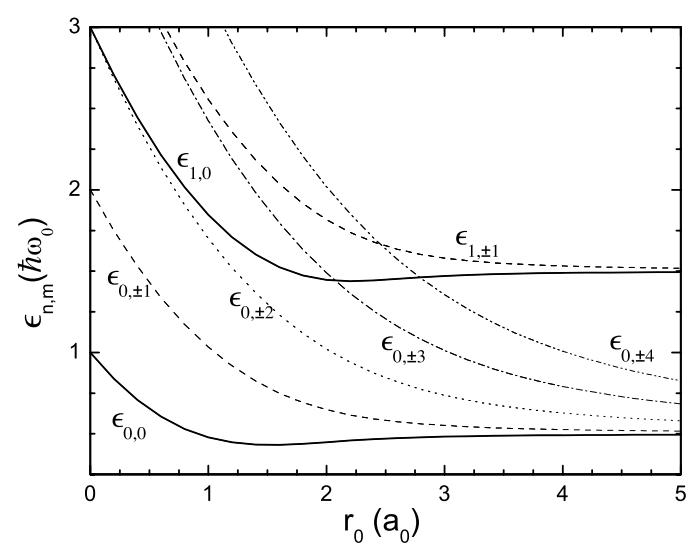

FIG. 1: Electron energy levels $\varepsilon_{n, m}$ in a single quantum ring as a function of the ring radius without electron-electron interaction.

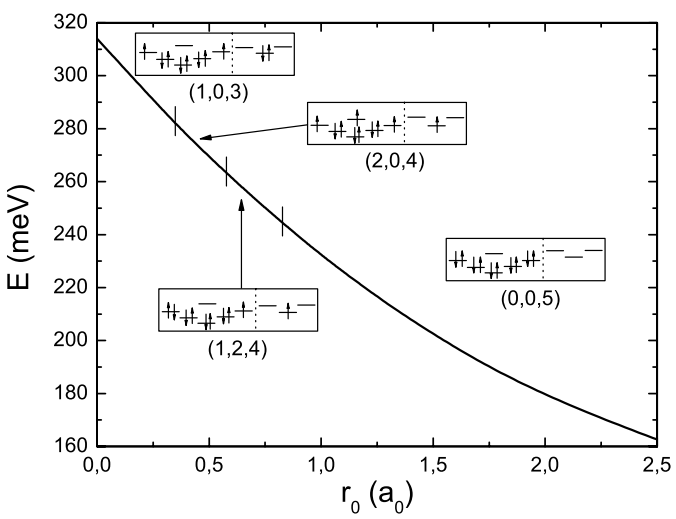

FIG. 2: The ground state energy for CQRs with 10 electrons as a function of ring radius for a fixed tunneling energy $\Delta=3.31 \mathrm{meV}$ and $\hbar \omega_{0}=5 \mathrm{meV}$. The inset indicates a single particle picture representation for each phase. The left (right) panel of the inset represents the bonding (antibonding) levels.

qualitatively by looking at the potential height $m^{*} \omega_{0}^{2} r_{0}^{2} / 2$ in the center of the ring.

In this work, we study theoretically the ground state configurations of two coupled quantum rings with 10 electrons. For two coupled quantum rings including electron-electron interaction, the ground state configurations can be labelled by three quantum numbers $\left(S_{z}, M_{z}, I_{z}\right)$ : total spin $S_{z}$, total angular momentum $M_{z}$ and the isospin quantum number $I_{z}$. The isospin quantum number is defined by the difference between the number of electrons in the bonding state and in the antibonding state divided by 2 .

We consider two coupled quantum rings of confinement energy $\hbar \omega_{0}=5 \mathrm{meV}$. The ground state energy and the corresponding electronic configuration of the system are determined by comparing the total energies for different possible configurations. The ground state configurations or phases are found for different inter-ring distance (tunneling strength) and 


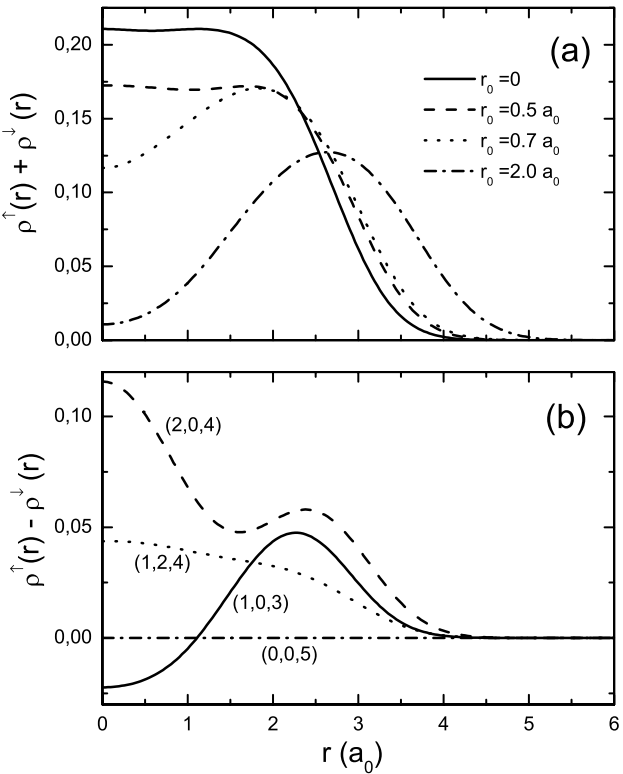

FIG. 3: (a) Total electron density distribution and (b) spin polarized electron density distribution for the CQRs with different ring radius $r_{0}=0,0.5,0.7$ and $2.0 \mathrm{a}_{0}$.

ring radii. In Fig. 2, we plot the ground state energy of the coupled quantum rings as a function of the ring radius for a fixed inter-ring distance $d=26 \AA$. Such an inter-ring distance corresponds to a tunneling energy $\Delta=3.31 \mathrm{meV}$. We found four distinct configurations (phases) for this tunneling strength. They are the configurations: $\left(S_{z}, M_{z}, I_{z}\right)=(1,0,3)$, $(2,0,4),(1,2,4)$ and $(0,0,5)$. The phase transitions occur at $r_{0}=0.32,0.58$, and $0.83 \mathrm{a}_{0}$. The insets in the figure indicate a single particle picture representation for each phase. The left and right panels of the inset, which are separated by the vertical dotted line, represent the bonding and antibonding levels, respectively. The phase $(1,0,3)$, for $r_{0}<0.32$, is consistent with the result obtained for two coupled quantum dots [4]. With increasing $r_{0}$, more electrons from the antibonding state transfer to the bonding state. We also notice that, the existence of the $(2,0,4)$ phase is a result of exchange effects. Although the single particle energy $\varepsilon_{0,2}$ is smaller than $\varepsilon_{1,0}$ as shown in Fig. 1, the $9^{\text {th }}$ electron in the bonding state occupies the state $(1,0)$ until this energy difference overcomes the exchange energy. For larger ring radius, all the electrons transfer to the bonding states. Fig. 3 illustrates (a) the total electron density and (b) the spin polarized electron density distributions for these four different phases. We identify two new phases originating from the quantum ring electronic structures as shown above. They are the $(1,2,4)$ and $(0,0,5)$ phases which have not been found for the case of vertically coupled dots. The convergency of the single particle levels $\varepsilon_{0, m}$ (for $\mathrm{m}=0, \pm 1, \ldots$ ) at large ring radius leads to the appearance of the two new phases.

Now, we investigate the effects of the inter-ring distance on the ground state phases for fixed ring radius. Fig. 4 shows
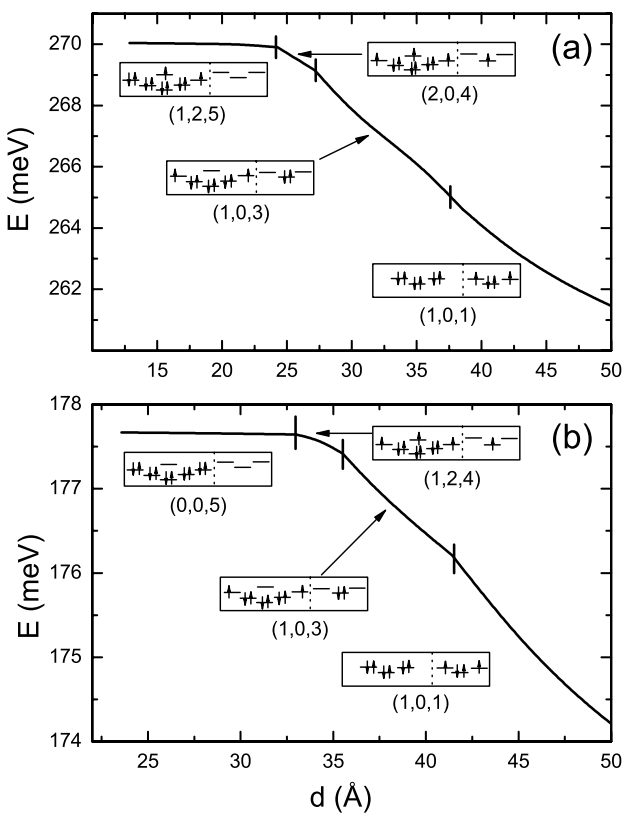

FIG. 4: Ground state energy for the CQRs as a function of the interring distance for different ring radii (a) $r_{0}=0.5 \mathrm{a}_{0}$ (b) $r_{0}=2.0 \mathrm{a}_{0}$.

the dependence of the ground state energy and phases of the coupled quantum rings on the inter-ring distance. For a small ring radius $r_{0}=0.5 \mathrm{a}_{0}$, as shown in Fig. 4(a), the ground state configurations are very similar to those of the coupled quantum dots. For larger ring radius of $r_{0}=2.0 \mathrm{a}_{0}$ in Fig. 4(b), at small inter-ring distance (large tunneling energy $\Delta$ ), all the electrons are in the bonding states. With increasing inter-ring distance, the energy $\Delta$ decreases. When it becomes smaller than the energy difference between the bonding levels $\left(\hbar \omega_{0}\right.$ in the single particle picture) the electron jumps to the lowest antibonding state and the ground state of the CQRs changes. Comparing the two figures, one can see that the same phases are found for CQDs and CQRs at large $d$. However, for small $d$, two new phases are found for the CQRs.

\section{CONCLUSIONS}

We have studied the ground state configurations of two vertically coupled quantum rings with tunneling between them. Within the spin density functional theory, the ground state energies and configurations are obtained for the system with 10 electrons as a function of the ring radius and the inter-ring distance. Our results indicate that two new phases, i.e., the $(1,2,4)$ and $(0,0,5)$ phases, show up in the strong tunneling regime for large ring radius.

\section{Acknowledgments}

The work was supported by FAPESP and CNPq, Brazil, and by Flemish Science Foundation (FWO-VL), Belgium. 
[1] A. Fuhrer, S. Lüscher, T. Ihn, T. Heinzel, K. Ensslin, W. Wegscheider, and M. Bichler, Nature (London) 413, 822 (2001); A. Lorke, R. J. Luyken, A. O. Govorov, J. P. Kotthaus, J. M. Garcia, and P. M. Petroff, Phys. Rev. Lett. 84, 2223 (2000).

[2] J. Simonin, C. R. Proetto, Z. Barticevic, and G. Fuster, Phys. Rev. B 70, 205305 (2004).

[3] D. Granados, J. M. Garcia, T. Ben, and S. I. Molina, Appl. Phys. Lett. 86, 071918 (2005).
[4] B. Partoens and F. M. Peeters, Phys. Rev. Lett. 84, 4433 (2000).

[5] M. Rotani, S. Amaha, K. Muraki, F. Manghi, E. Molinari, S. Tarucha, and D. G. Austing, Phys. Rev. B. 69, 085327 (2004).

[6] J. C. Lin and G. Y. Guo, Phy. Rev. B. 65, 035304 (2001).

[7] B. Tanatar and D. M. Ceperley, Phys. Rev. B 39, 5005 (1989).

[8] V. Fock, Z. Phys. 47, 446 (1928).

[9] C. Darwin, Proc. Cambridge Philos. Soc. 27, 86 (1930). 